\title{
Two collection formulas
}

\author{
Egorychev G.P., Kolesnikov S.G., Leontiev V.M.
}

\begin{abstract}
Let $G$ be a group, $x, y \in G$ and $H$ is a normal closure in $G$ of the subgroup generated by all commutators of $x, y$ that include more than two occurrences of $y$. Our main result is a parametrization of the uncollected part of Hall's collection formula for $(x y)^{n}$ and two different collection formulas modulo $H$ in an explicit form. In particular, we found explicit expressions for the exponents of the commutators in the Hall's collection formula for both known cases and some unknown cases. Also we give combinatorial identities, which simplify those expressions and help to investigate their divisibility.
\end{abstract}

Keywords: collection process, binary weight of an integer, the method of coefficients.

\section{Introduction and main results}

In 1932, P. Hall proved a formula in [5] that expresses the product $(x y)^{n}$ in terms of $x^{n}$, $y^{n}$ and commutators of $x$ and $y$. The formula, later called the Hall's collection formula, led to new directions of the study of $p$-groups. The subsets $\Omega_{i}$ and $\mho_{j}$ of the $p$-group $G$, formed respectively by $p^{i}$-th powers and elements whose orders divide $p^{j}$, arose in a natural way and became actively used. Investigation of conditions under which $\Omega_{i}$ and $\mho_{j}$ are subgroups of $G$ (if $G$ is an abelian group, it is obvious) led, in particular, to the notion of regular $p$-groups. The relations between $\Omega_{i}, \mho_{j}$ and the subgroups generated by commutators are often used in solving $p$-group theory problems and also became the basis of the notion of powerful $p$-groups.

Let us recall P. Hall's result. For any two elements $x, y$ of any group $\mathrm{G}$ let the formally distinct complex commutators $R_{1}, R_{2}, \ldots, R_{i}, \ldots$ of $x, y$ be arranged in order of increasing weights (the order among the commutators of the same weight is arbitrary). Then there exists a series of integer-valued polynomials $f_{1}(n), f_{2}(n), \ldots, f_{i}(n), \ldots$ all vanishing for $n=0$ and such that

$$
(x y)^{n}=R_{1}^{f_{1}(n)} R_{2}^{f_{2}(n)} \ldots R_{i}^{f_{i}(n)} \ldots,
$$

where $f_{1}(n)=f_{2}(n)=n$ and the degree of $f_{i}(n)$ does not depend on $n$. It is a difficult problem to find an explicit form of the polynomial $f_{i}(n)$ in general case.

It is noted in $\left[4\right.$, p. 326] that the formula (1) includes the commutator $\left[y,{ }_{u} x\right]$ to the power of $\left(\begin{array}{c}n \\ u+1\end{array}\right)$. Here and below we denote by $\left(\begin{array}{c}m \\ k\end{array}\right)$ the binomial coefficient (the number of combinations of $k$ items from a set of $m$ items). By definition, we put $\left[y,{ }_{0} x\right]=y$ and $\left[y,{ }_{u} x\right]=\left[\left[y,{ }_{u-1} x\right], x\right]$ for integer $u>0$. Also we put $\left[y,{ }_{u} x,{ }_{v} y\right]=\left[\left[y,{ }_{u} x\right],{ }_{v} y\right]$ for integers $u \geqslant 0, v \geqslant 0$. In the article [6] (see also [9]), devoted to the investigation of Burnside problem for exponent 8, the following expressions for the exponents of the commutators $\left[y,{ }_{u} x,{ }_{v} y\right](u \geqslant 1, v \geqslant 0),\left[\left[y,{ }_{u} x,{ }_{v} y\right],[y, x]\right]$ $(u \geqslant 2$ and $v=0$, or $u, v \geqslant 1),[[y, x, y],[y, x, x]]$ from $(1)$, respectively, were found:

$$
\sum_{k=1}^{n-1}\left(\begin{array}{l}
k \\
u
\end{array}\right)\left(\begin{array}{l}
k \\
v
\end{array}\right), \quad \sum_{k=1}^{n-1}\left(\begin{array}{l}
k \\
v
\end{array}\right)\left[\left(\begin{array}{l}
k \\
2
\end{array}\right)\left(\begin{array}{l}
k \\
u
\end{array}\right)+\left(\begin{array}{c}
k \\
u+1
\end{array}\right)\right], \quad \sum_{k=0}^{n-1} k\left[2\left(\begin{array}{l}
k \\
2
\end{array}\right)+(k+2)\left(\begin{array}{l}
k \\
3
\end{array}\right)\right] .
$$

On the other hand, it is possible to get the collection formulas in an explicit form by imposing reasonable conditions on the group $G$. For example, if the commutator $[y, x]$ belongs to the center of $G$, then for any natural number $n$ we have

$$
(x y)^{n}=x^{n} y^{n}[y, x]^{\left(\begin{array}{c}
n \\
2
\end{array}\right)} .
$$


If the subgroup, generated by an element $y$ and the commutator subgroup $G^{\prime}=\Gamma_{2}(G)=[G, G]$, is abelian, then

$$
(x y)^{n}=x^{n} y^{n}[y, x]^{\left(\begin{array}{l}
n \\
2
\end{array}\right)}\left[y,{ }_{2} x\right]^{\left(\begin{array}{l}
n \\
3
\end{array}\right)} \ldots\left[y,{ }_{n-1} x\right]^{\left(\begin{array}{l}
n \\
n
\end{array}\right)} .
$$

Imposing weaker conditions than in (2) and (3), A.I. Skopin proved the following results in [8]. Suppose $G$ is a group. If $\left[\Gamma_{3}(G), \Gamma_{3}(G)\right]=1$ (here $\Gamma_{3}(G)=\left[\Gamma_{2}(G), G\right]$ ), then for any $x \in G$ and $y \in G^{\prime}$

$$
(x y)^{n}=x^{n} y^{n} \prod_{i=1}^{n-1} \prod_{j=0}^{n-1}\left[y,{ }_{i} x,{ }_{j} y\right]^{\sum_{k=1}^{n-1}\left(\begin{array}{c}
k \\
i
\end{array}\right)\left(\begin{array}{c}
k \\
j
\end{array}\right)},
$$

and if $\left[\Gamma_{2}(G), \Gamma_{2}(G), \Gamma_{2}(G)\right]=1$, then

$$
(x y)^{n}=x^{n} y^{n} \prod_{i=1}^{n-1}\left[y,{ }_{i} x\right]{ }^{\left({ }_{i+1}\right)} \prod_{i=0}^{n-2} \prod_{j=i+1}^{n-1}\left[\left[y,{ }_{i} x\right],\left[y,{ }_{j} y\right]\right]^{c_{i j}^{n}},
$$

where the coefficients $c_{i j}^{n}$ are determined by recurrence relations (explicit expressions for $c_{i j}^{n}$ were not found).

In this paper we propose a way to parametrize the uncollected part of the Hall's collection formula. It helps to compute the exponents of the commutators in (1) for known cases and for new ones and to get new collection formulas. Now we introduce the aforementioned parametrization and consider our main results.

Let $G$ be a group, $x, y \in G$. By collecting $x$ and $y$ in the product $(x y)^{2}$, we have $(x y)^{2}=$ $x^{2} y^{2} R_{2}$, where $R_{2}=[y, x][y, x, y]$. Further, we represent the product $(x y)^{3}$ in the form $(x y)^{3}=$ $x y(x y)^{2}=x y x^{2} y^{2} R_{2}$ and also collect $x$ and $y$. After collecting $x$, we parametrize the occurrences of $x$ in the resulting commutators by the function $\omega$ with integral argument

$$
(x y)^{3}=x^{3} y[y, x][y, x]\left[y,{ }_{2} x\right] y^{2} R_{2}=x^{3} y\left(\prod_{i=1}^{3}[y, \omega(i) x]\right) y^{2} R_{2},
$$

where $\omega(1)=1, \omega(2)=1, \omega(3)=2$. Continuing this line of reasoning for $y$, we get

$$
(x y)^{3}=x^{3} y^{3}\left(\prod_{i=1}^{3}[y, \omega(i) x]\left(\prod_{j=1}^{3}[y, \omega(i) x, \omega(j) y]\right)\right) R_{2} .
$$

By putting $\omega(0)=0$ and recalling that $\left[y,{ }_{0} x\right]=y$, we represent the formula (6) in a more convenient form (where $R_{2}$ is parametrized similarly)

$$
(x y)^{3}=x^{3} y^{3}\left(\prod_{i=1}^{3}\left(\prod_{j=0}^{3}[y, \omega(i) x, \omega(j) y]\right)\right)\left(\prod_{i=1}^{1}\left(\prod_{j=0}^{1}[y, \omega(i) x, \omega(j) y]\right)\right) .
$$

We note that the function $\omega(i)$ is equal to the binary weight of the number $i$. In the sequel, we put that $\omega(i)$ is equal to the count of units in the binary representation of the non-negative integer $i$. The following statement holds.

Theorem 1. Let $G$ be a group, $x, y \in G$. Then for any $n \in \mathbb{N}$ we have

$$
(x y)^{n}=x^{n} y^{n} \prod_{k=1}^{n-1} \prod_{i=1}^{2^{n-k}-1} \prod_{j=0}^{2^{n-k}-1}\left[y, \omega(i) x,{ }_{\omega(j)} y\right] .
$$


Remark 1. If we write $n$-multiple non-commutative product with variable indices $i_{1}, \ldots, i_{n}$, then the multiplier corresponding to the set $\left(i_{1}^{\prime}, \ldots, i_{n}^{\prime}\right)$ follows after the multiplier that corresponds to the set $\left(i_{1}^{\prime \prime}, \ldots, i_{n}^{\prime \prime}\right)$ if and only if $\left(i_{1}^{\prime \prime}, \ldots, i_{n}^{\prime \prime}\right)$ is less than $\left(i_{1}^{\prime}, \ldots, i_{n}^{\prime}\right)$ with respect to the lexicographic order. Besides, if the upper bound of summation (multiplication) is less than the lower bound, then we put the sum (product) is equal to zero (one).

For every $n \in \mathbb{N}, u \in \mathbb{N}, v \in \mathbb{N}_{0}$, the following lemma shows an explicit formula for the number $E_{n}(u, v)$ of the commutator $\left[y,{ }_{u} x,{ }_{v} y\right]$, in the product

$$
P_{n}=\prod_{k=1}^{n-1} \prod_{i=1}^{2^{n-k}-1} \prod_{j=0}^{2^{n-k}-1}[y, \omega(i) x, \omega(j) y]
$$

Lemma 1. Suppose $n, u \in \mathbb{N}$ and $v \in \mathbb{N}_{0}$. Then

$$
E_{n}(u, v)=\sum_{m=1}^{n-1}\left(\begin{array}{c}
m \\
u
\end{array}\right)\left(\begin{array}{c}
m \\
v
\end{array}\right)
$$

Some collection formulas mentioned above follow easily from Theorem 1 and Lemma 1. Indeed, if the subgroup $\left\langle y, G^{\prime}\right\rangle$ of the group $G$ is abelian, then, firstly, all commutators $\left[y,{ }_{u} x,{ }_{v} y\right]$, $u \geqslant 1, v \geqslant 1$, are equal to one, secondly, commutators of the form $\left[y,{ }_{u} x\right], u \geqslant 1$, commute with each other, thirdly, by Lemma 1 , the quantity of $\left[y,{ }_{u} x\right]$ in the product $P_{n}$ is equal to

$$
E_{n}(u, 0)=\sum_{k=1}^{n-1}\left(\begin{array}{l}
k \\
u
\end{array}\right)\left(\begin{array}{l}
k \\
0
\end{array}\right)=\sum_{k=1}^{n-1}\left(\begin{array}{l}
k \\
u
\end{array}\right)=\left(\begin{array}{c}
n \\
u+1
\end{array}\right)
$$

Thus, we get (3).

If $x \in G, y \in \Gamma_{2}(G)$ and $\left[\Gamma_{3}(G), \Gamma_{3}(G)\right]=1$, then all commutators of the form $\left[y,{ }_{u} x,{ }_{v} y\right]$, $u \geqslant 1, v \geqslant 0$, commute with each other. Therefore,

$$
(x y)^{n}=x^{n} y^{n} \prod_{u=1}^{n-1} \prod_{v=0}^{n-1}\left[y,{ }_{u} x,{ }_{v} y\right]^{E_{n}(u, v)}=x^{n} y^{n} \prod_{u=1}^{n-1} \prod_{v=0}^{n-1}\left[y,{ }_{u} x,{ }_{v} y\right]^{\sum_{k=1}^{n-1}\left(\begin{array}{l}
k \\
u
\end{array}\right)\left(\begin{array}{l}
k \\
v
\end{array}\right)} .
$$

Let us return to the identity (7) and compute the number of the commutators $\left[\left[y,{ }_{u} x\right],\left[y,{ }_{v} x\right]\right]$, which arise in the process of collecting $\left[y,{ }_{v} x\right]$ in $P_{n}$. It is clear that the commutator $\left[\left[y,{ }_{u} x\right],\left[y,{ }_{v} x\right]\right]$ arises every time when consecutive elements $\left[y,{ }_{u} x\right]\left[y,{ }_{v} x\right]$ change places. Suppose that the commutator $\left[y,{ }_{v} x\right]$ corresponds to the indices $k=k_{0}, i=i_{0}, j=0$ in the product $P_{n}$, where $1 \leqslant k_{0} \leqslant n-1,1 \leqslant i_{0} \leqslant 2^{n-k_{0}}-1, \omega\left(i_{0}\right)=v$. Let us find the number of $\left[y,{ }_{u} x\right]$ to the left of $\left[y, \omega\left(i_{0}\right) x\right]$. By representing $P_{n}$ in the form

$$
P_{n}=\left(\prod_{k=1}^{k_{0}-1} \prod_{i=1}^{2^{n-k}-1} \prod_{j=0}^{2^{n-k}-1}[y, \omega(i) x, \omega(j) y]\right)\left(\prod_{i=1}^{i_{0}-1} \prod_{j=0}^{2^{n-k_{0}}-1}[y, \omega(i) x, \omega(j) y]\right)\left[y, \omega\left(i_{0}\right) x\right] \ldots
$$

we see that the number of $\left[y,{ }_{u} x\right]$ in the second product is equal to the number of solutions of the equation $\omega(i)=u$ with respect to $i, i \in\left[1, i_{0}-1\right]$. In the first product, it is equal to the number of solutions of the equation $\omega(i)=u$ for $i \in\left[1,2^{n-k}-1\right]$ and for every $k$ from 1 to $k_{0}-1$. In view of this, for any $q, x \in \mathbb{Z}$, we put

$$
W(q, x)=\{i \mid \omega(i)=x, 0 \leqslant i \leqslant q\} .
$$


Also, for any $q, m \in \mathbb{N}$ such that $1 \leqslant m \leqslant \omega(q)$, by $L(q, m)$, we denote the position's number of the $m$-th unit in the base 2 representation of $q$ (we count units from left to right and, as usual, enumerate digit positions from right to left, starting from zero). For example, $L(41,1)=$ $L\left(101001_{2}, 1\right)=5, L\left(101001_{2}, 2\right)=3, L\left(101001_{2}, 3\right)=0$.

In the following theorem we compute the cardinality of the set $W(q, x)$ and necessary sums of such cardinalities.

Theorem 2. Suppose $m, q, x \in \mathbb{Z}$. 1) If $q<0$ or $x<0$, then $|W(q, x)|=0$. 2) If $m \geqslant 0$, $x \geqslant 0$, then $\left|W\left(2^{m}-1, x\right)\right|=\left(\begin{array}{c}m \\ x\end{array}\right)$. 3) If $q \geqslant 0, x \geqslant 0$, then

$$
|W(q, x)|=\sum_{k=1}^{\omega(q)}\left(\begin{array}{c}
L(q, k) \\
x-k+1
\end{array}\right)+\delta_{\omega(q), x}
$$

where $\delta_{i, j}$ is the Kronecker delta. 4) If $u, v \in \mathbb{Z}$ and $m \geqslant u \geqslant 0, m \geqslant v \geqslant 0$, then

$$
\sum_{j \in W\left(2^{m}-1, v\right)}|W(j-1, u)|=\sum_{k=1}^{v} \sum_{i=v-k}^{m-k}\left(\begin{array}{c}
i \\
u-k+1
\end{array}\right)\left(\begin{array}{c}
m-i-1 \\
k-1
\end{array}\right)\left(\begin{array}{c}
i \\
v-k
\end{array}\right) .
$$

Theorem 2 helps us to compute the exponents of the commutators $\left[\left[y,{ }_{u} x,\right],\left[y,{ }_{v} x\right]\right], 1 \leqslant v<$ $u \leqslant n-1$, in the Hall's collection formula and find an explicit form of this formula modulo $H$, where $H$ is a normal closure of the subgroup generated by all commutators of $x, y$ that includes more than two occurrences of $y$.

Theorem 3. Suppose $G$ is a group, $x, y \in G$, the subgroup $H$ is defined as above. Then for any $n \in \mathbb{N}$ we have

$$
(x y)^{n}=x^{n} y^{n} \prod_{u=1}^{n-1}\left[y,{ }_{u} x\right]^{\left(\begin{array}{c}
n \\
u+1
\end{array}\right)} \prod_{u=1}^{n-1}\left[y,{ }_{u} x, y\right]^{n\left(\begin{array}{c}
n \\
u+1
\end{array}\right)-\left(\begin{array}{c}
n+1 \\
u+2
\end{array}\right)} \prod_{n-1 \geqslant u>v \geqslant 1}\left[\left[y,{ }_{u} x\right],\left[y,{ }_{v} x\right]\right]^{f_{n}(u, v)}(\bmod H),
$$

where

$$
f_{n}(u, v)=\sum_{m=1}^{n-1} \sum_{k=1}^{v} \sum_{i=v-k}^{n-m-k}\left(\begin{array}{c}
i \\
u-k+1
\end{array}\right)\left(\begin{array}{c}
n-m-i-1 \\
k-1
\end{array}\right)\left(\begin{array}{c}
i \\
v-k
\end{array}\right)+\sum_{m=1}^{n-2} \sum_{k=m+1}^{n-1}\left(\begin{array}{l}
m \\
v
\end{array}\right)\left(\begin{array}{l}
k \\
u
\end{array}\right) .
$$

In the following theorem we obtain the formula for $(x y)^{n}$ by recursive collection process under the condition $H=1$. The recursion means that we apply the collection process to $(x y)^{k}=(x y)^{k-1} x y$ after the end of the collection process for $(x y)^{k-1}$ (as, for example, in the proofs of (2) and (3)).

Theorem 4. Suppose $G$ is a group, $x, y \in G, H$ is defined as above. If $H=1$, then, for any $n \in \mathbb{N}$, we have

$$
(x y)^{n}=x^{n} y^{n} \prod_{i>0}\left[y,{ }_{i} x\right]^{\alpha_{n}(i)} \prod_{i>0, j \geqslant 0}\left[y,{ }_{i} x, y,{ }_{j} x\right]^{\beta_{n}(i, j)} \prod_{i>0, k \geqslant 0}\left[\left[y,{ }_{i+1} x\right],\left[y,{ }_{i} x\right],{ }_{k} x\right]^{\mu_{n}(i, k)} .
$$

Here $\alpha_{n}(i)=\left(\begin{array}{c}n \\ i+1\end{array}\right), \beta_{n}(1, j)=\left(\begin{array}{c}n \\ j+3\end{array}\right)+\left(\begin{array}{c}n+1 \\ j+3\end{array}\right)$ and $\beta_{n}(i, j)=\left(\begin{array}{c}n+1 \\ i+j+2\end{array}\right)$, when $i>1$. And, finally,

$$
\mu_{n}(i, 0)=\theta_{n}(i)=\sum_{m=1}^{n-1}\left(\begin{array}{c}
\alpha_{m}(i) \\
2
\end{array}\right), \quad \mu_{n}(i, k)=\sum_{s_{k}=1}^{n-k-1} \sum_{s_{k-1}=1}^{s_{k}} \ldots \sum_{s_{1}=1}^{s_{2}} \sum_{m=1}^{s_{1}}\left(\begin{array}{c}
\alpha_{m}(i) \\
2
\end{array}\right), k \geqslant 1 .
$$


Remark 2. In the formula (12), the multipliers' order in the first product is important. The order in other products can be arbitrary because all commutators in these products commute with each other.

Further, we use the Egorychev method of coefficients ([1]-[2]), some well-known facts from the theory of residues in complex analysis and elementary properties of binomial coefficient to reduce to a 1-multiple sum the following $r$-multiple combinatorial sum with linear constrains on the summation indices (Theorem 5):

$$
\sum_{s_{r-1}=1}^{n} \sum_{s_{r-2}=1}^{s_{r-1}} \ldots \sum_{s_{1}=1}^{s_{2}} \sum_{s=1}^{s_{1}}\left(\begin{array}{c}
s \\
i+1
\end{array}\right)^{m}, r, n, m \in \mathbb{N}, i \in \mathbb{N}_{0} .
$$

As a consequence, the numbers $\mu_{n}(t, k)$ are expressed as a 1-multiple sum.

Let us note that Theorems 3-5 were announced in [3]. The results in Theorems 1 and 2 were presented at the August Möbius Contest in 2016 [7].

\section{Proofs}

The sequence $\{\omega(i)\}_{i=0}^{\infty}$ satisfies the recurrence relations: $\omega(0)=0, \omega(2 i)=\omega(i), \omega(2 i+1)=$ $\omega(i)+1$. So, for any non-negative integer $m$ we have

$$
\begin{aligned}
\left(\prod_{i=0}^{2^{m}-1}[r, \omega(i) z]\right) z & =z \prod_{i=0}^{2^{m}-1}[r, \omega(i) z][r, \omega(i)+1 z]= \\
& =z \prod_{i=0}^{2^{m}-1}[r, \omega(2 i) z][r, \omega(2 i+1) z]=z \prod_{i=0}^{2^{m+1}-1}[r, \omega(i) z] .
\end{aligned}
$$

Using $2 n$ times the identity

$$
\left(\prod_{i=0}^{2^{m}-1}[r, \omega(i) z]\right) z=z \prod_{i=0}^{2^{m+1}-1}[r, \omega(i) z]
$$

we collect $x$ and $y$ in $x y x^{n} y^{n}$ as follows:

$$
\begin{aligned}
x y x^{n} y^{n} & =x\left(\prod_{i=0}^{2^{0}-1}[y, \omega(i) x]\right) x^{n} y^{n}=x^{n+1}\left(\prod_{i=0}^{2^{n}-1}[y, \omega(i) x]\right) y^{n}= \\
& =x^{n+1} y\left(\prod_{i=1}^{2^{n}-1} \prod_{j=0}^{2^{0}-1}[y, \omega(i) x, \omega(j) y]\right) y^{n}=x^{n+1} y^{n+1} \prod_{i=1}^{2^{n}-1} \prod_{j=0}^{2^{n}-1}[y, \omega(i) x, \omega(j) y] .
\end{aligned}
$$

Thus,

$$
x y x^{n} y^{n}=x^{n+1} y^{n+1} \prod_{i=1}^{2^{n}-1} \prod_{j=0}^{2^{n}-1}[y, \omega(i) x, \omega(j) y] .
$$


Using induction on $n$ and the identity (13), we have

$$
\begin{aligned}
(x y)^{n+1} & =x y(x y)^{n}=x y x^{n} y^{n} \prod_{k=1}^{n-1} \prod_{i=1}^{2^{n-k}-1} \prod_{j=0}^{2^{n-k}-1}[y, \omega(i) x, \omega(j) y]= \\
& =x^{n+1} y^{n+1}\left(\prod_{i=1}^{2^{n}-1} \prod_{j=0}^{2^{n}-1}[y, \omega(i) x, \omega(j) y]\right) \prod_{k=1}^{n-1} \prod_{i=1}^{2^{n-k}-1} \prod_{j=0}^{2^{n-k}-1}[y, \omega(i) x, \omega(j) y]= \\
& =x^{n+1} y^{n+1} \prod_{k=0}^{n-1} \prod_{i=1}^{2^{n-k}-1} \prod_{j=0}^{2^{n-k}-1}[y, \omega(i) x, \omega(j) y]= \\
& =x^{n+1} y^{n+1} \prod_{k=1}^{n} \prod_{i=1}^{2^{n-k+1}-1} \prod_{j=0}^{2^{n-k+1}-1}[y, \omega(i) x, \omega(j) y] .
\end{aligned}
$$

Theorem 1 is proved.

For proving Lemma 1, we fix $n, u, v$ and denote by $E_{n, k}(u, v)$ the number of the commutators $\left[y,{ }_{u} x,{ }_{v} y\right]$ in the product

$$
P_{n, k}(x, y)=\prod_{i=1}^{2^{n-k}} \prod_{j=0}^{2^{n-k}-1}[y, \omega(i) x, \omega(j) y]
$$

where $k$ is fixed, $1 \leqslant k \leqslant n-1$. It is easy to see that $E_{n, k}(u, v)$ is equal to the number of such integers $i$ that $1 \leqslant i \leqslant 2^{n-k}-1, \omega(i)=u$, multiplied by the number of such integers $j$ that $0 \leqslant j \leqslant 2^{n-k}-1, \omega(j)=v$. Since we need no more than $n-k$ digit positions for the binary representation of integer $i \in\left[1,2^{n-k}-1\right]$, the number of such integers with the condition $\omega(i)=u$ is equal to the number of ways to place $u$ units in $n-k$ positions, i.e. $\left(\begin{array}{c}n-k \\ u\end{array}\right)$. Similarly, the number of such integers $j$ that $j \in\left[0,2^{n-k}-1\right], \omega(j)=v$ is equal to $\left(\begin{array}{c}n-k \\ v\end{array}\right)$ (for $j=0$ we have $\omega(0)=0$ and the number of integers with zero units is equal to $\left.1=\left(\begin{array}{c}n-k \\ 0\end{array}\right)\right)$. Therefore,

$$
E_{n, k}(u, v)=\left(\begin{array}{c}
n-k \\
u
\end{array}\right)\left(\begin{array}{c}
n-k \\
v
\end{array}\right)
$$

Note that if $u>n-k$ or $v>n-k$, then $P_{n, k}(u, v)$ does not contain the commutator $\left[y,{ }_{u} x,{ }_{v} y\right]$ and at the same time $\left(\begin{array}{c}n-k \\ u\end{array}\right)=0$ or $\left(\begin{array}{c}n-k \\ v\end{array}\right)=0$. Hence, the equality (15) holds for any integers $u \geqslant 1$ and $v \geqslant 0$. To conclude the proof, it remains to note that

$$
E_{n}(u, v)=\sum_{k=1}^{n-1} E_{n, k}(u, v)=\sum_{k=1}^{n-1}\left(\begin{array}{c}
n-k \\
u
\end{array}\right)\left(\begin{array}{c}
n-k \\
v
\end{array}\right)=\sum_{k=1}^{n-1}\left(\begin{array}{l}
k \\
u
\end{array}\right)\left(\begin{array}{l}
k \\
v
\end{array}\right)
$$

Lemma 1 is proved.

Let us prove Theorem 2. It is clear that the set $W(q, x)$ is empty when $q<0$ or $x<0$. Furthermore, the cardinality of the set $W\left(2^{m}-1, x\right)$, when $m \geqslant 0, x \geqslant 0$, is equal to the number of ways to place $x$ units in $m$ positions (we need no more than $m$ digit positions for the binary representation of integer $\left.i \in\left[0,2^{m}-1\right]\right)$, therefore,

$$
\left|W\left(2^{m}-1, x\right)\right|=\sum_{j \in W\left(2^{m}-1, x\right)} 1=\left(\begin{array}{c}
m \\
x
\end{array}\right) .
$$


Finally, if $q \in W(q, x)$, then $|W(q-1, x)|=|W(q, x)|-1$, and if $q \notin W(q, x)$, then $|W(q-1, x)|=$ $|W(q, x)|$. So, for any non-negative integers $q$ and $x$ we have

$$
|W(q-1, x)|=|W(q, x)|-\delta_{\omega(q), x} .
$$

Further, when $q=0$ and $x \geqslant 0$, we have

$$
|W(0, x)|=\sum_{k=1}^{0}\left(\begin{array}{c}
L(q, k) \\
x-k+1
\end{array}\right)+\delta_{0, x}=\delta_{0, x}
$$

Now suppose $q \geqslant 1, x \geqslant 0$. For the binary representation of $q$ we need $L(q, 1)+1$ digit positions. Moreover, the $j$-th digit of $q$ is one if and only if $j=L(q, t)$ for some $t, 1 \leqslant t \leqslant \omega(q)$. Therefore, $|W(q, x)|$ is equal to the number of ways to place $x$ units in $L(q, 1)+1$ positions under the condition that resulting numbers do not exceed $q$. We can get any non-negative integer that does not exceed $q-1$ and has $x$ units in its binary representation by changing the $L(q, t)$-th unit of $q, 1 \leqslant t \leqslant \omega(q)$, by zero and placing $x-t+1$ units in the lower positions in any way. Thus, the number of integers from 0 to $q-1$ with $x$ units in the binary representation is equal to the sum

$$
\sum_{k=1}^{\omega(q)}\left(\begin{array}{c}
L(q, k) \\
x-k+1
\end{array}\right)
$$

Adding the number $\delta_{\omega(q), x}$, which is equal to one if $\omega(q)=x$ and zero otherwise, we get (9).

Note the following obvious equality:

$$
L(q, m)=\left\lfloor\log _{2}\left(q-\sum_{k=1}^{m-1} 2^{L(q, k)}\right)\right\rfloor
$$

here $\lfloor\cdot\rfloor$ is the floor function.

Let us prove assertion 4) of Theorem 2. To transform the left-hand side of (10), we use the formula (17), assertion 3) of the theorem, and then we change the order of summation,

$$
\sum_{j \in W\left(2^{m}-1, v\right)}|W(j-1, u)|=\sum_{j \in W\left(2^{m}-1, v\right)} \sum_{k=1}^{\omega(j)}\left(\begin{array}{c}
L(j, k) \\
u-k+1
\end{array}\right)=\sum_{k=1}^{v} \sum_{j \in W\left(2^{m}-1, v\right)}\left(\begin{array}{c}
L(j, k) \\
u-k+1
\end{array}\right) .
$$

Suppose $v \geqslant 1$. We fix the index $k$ and collect terms with equal values of $L(j, k)$ in the sum

$$
\sum_{j \in W\left(2^{m}-1, v\right)}\left(\begin{array}{c}
L(j, k) \\
u-k+1
\end{array}\right) .
$$

For the binary representation of any integer from 0 to $2^{m}-1$ we need no more than $m$ digit positions. So, the $k$-th unit in the binary representation of any $j \in W\left(2^{m}-1, v\right)$ can not be placed, firstly, to the left of the $(m-k)$-th position (otherwise we need more than $m$ digit positions for the binary representation of $j$ ), secondly, to the right of the $(v-k)$-th position (otherwise the number of units in the binary representation of $j$ is less than $v$ ). Thus, for any $j \in W\left(2^{m}-1, v\right)$ we have $v-k \leqslant L(j, k) \leqslant m-k$. Furthermore, for any $i \in[v-k, m-k]$ there exists a number $j \in W\left(2^{m}-1, v\right)$, for example, $j=0 \ldots 01_{i+k-1} \ldots 1_{i} \ldots 1_{i-v+k} 0 \ldots 0$, such that $L(j, k)=i$. The number of all $j$ such that $L(j, k)=i$ is equal to the product of the number of 
ways to place $k-1$ units in $m-1-i$ positions to the left of $i$-th position and the number of ways to place $v-k$ units in $i$ positions to the right of $i$-th position, i.e. $\left(\begin{array}{c}m-i-1 \\ k-1\end{array}\right)\left(\begin{array}{c}i \\ v-k\end{array}\right)$. Thus,

$$
\sum_{k=1}^{v} \sum_{j \in W\left(2^{m}-1, v\right)}\left(\begin{array}{c}
L(j, k) \\
u-k+1
\end{array}\right)=\sum_{k=1}^{v} \sum_{i=v-k}^{m-k}\left(\begin{array}{c}
i \\
u-k+1
\end{array}\right)\left(\begin{array}{c}
m-i-1 \\
k-1
\end{array}\right)\left(\begin{array}{c}
i \\
v-k
\end{array}\right) .
$$

Theorem 2 is proved.

Proving Theorem 3. By Lemma 1, the number of the commutators $\left[y,{ }_{u} x\right], u \geqslant 1$, in the identity $(7)$ is equal to $E_{n}(u, 0)=\left(\begin{array}{c}n \\ u+1\end{array}\right)$, and the number of $\left[y,{ }_{u} x, y\right], u \geqslant 1$, is equal to

$$
E_{n}(u, 1)=\sum_{k=1}^{n-1}\left(\begin{array}{l}
k \\
u
\end{array}\right)\left(\begin{array}{l}
k \\
1
\end{array}\right)=\sum_{k=1}^{n-1} k\left(\begin{array}{l}
k \\
u
\end{array}\right)=n\left(\begin{array}{c}
n \\
u+1
\end{array}\right)-\left(\begin{array}{l}
n+1 \\
u+2
\end{array}\right) .
$$

We collect commutators in the identity $(7)$ as follows: first we collect $[y, x]$, then $\left[y,{ }_{2} x\right], \ldots$, after all commutators of the form $\left[y,{ }_{i} x\right]$ are collected we collect $[y, x, y],\left[y,{ }_{2} x, y\right], \ldots$ and so on. From definition of the subgroup $H$ it follows that all commutators arising in the collection process belong to $H$ except $\left[\left[y,{ }_{u} x\right],\left[y,{ }_{v} x\right]\right], n-1 \geqslant u>v \geqslant 1$ (because $\left[y,{ }_{v} x\right]$ is collected before $\left.\left[y,{ }_{u} x\right]\right)$. Thus we have

$$
(x y)^{n}=x^{n} y^{n} \prod_{u=1}^{n-1}\left[y,{ }_{u} x\right]^{\left(\begin{array}{c}
n \\
u+1
\end{array}\right)} \prod_{u=1}^{n-1}\left[y,{ }_{u} x, y\right]^{n\left(\begin{array}{c}
n \\
u+1
\end{array}\right)-\left(\begin{array}{c}
n+1 \\
u+2
\end{array}\right)} \prod_{n-1 \geqslant u>v \geqslant 1}\left[\left[y,{ }_{u} x\right],\left[y,{ }_{v} x\right]\right]^{f_{n}(u, v)}(\bmod H) .
$$

To conclude the proof, it remains to find $f_{n}(u, v)$.

It is clear that the commutator $\left[\left[y,{ }_{u} x\right],\left[y,{ }_{v} x\right]\right]$ arises every time when consecutive elements $\left[y,{ }_{u} x\right]\left[y,{ }_{v} x\right]$ change places. So, let us fix $u$ and $v$ such that $n-1 \geqslant u>v \geqslant 1$ and consider in detail the process of collecting the commutators $\left[y,{ }_{v} x\right]$ in the product $P_{n}$. Suppose that the commutator $\left[y,{ }_{v} x\right]$ corresponds to the indices $k=k_{0}, i=i_{0}, j=0$ in $P_{n}$, where $1 \leqslant k_{0} \leqslant n-1$, $i_{0} \in W\left(2^{n-k_{0}}-1, v\right)$. Let us find the number of $\left[y,{ }_{u} x\right]$ to the left of $\left[y,{ }_{\omega\left(i_{0}\right)} x\right]$. By representing $P_{n}$ in the form

$$
P_{n}=\left(\prod_{k=1}^{k_{0}-1} \prod_{i=1}^{2^{n-k}-1} \prod_{j=0}^{2^{n-k}-1}\left[y, \omega_{(i)} x, \omega(j) y\right]\right)\left(\prod_{i=1}^{i_{0}-1} \prod_{j=0}^{2^{n-k_{0}}-1}\left[y, \omega_{\omega(i)} x, \omega_{\omega(j)} y\right]\right)\left[y, \omega\left(i_{0}\right) x\right] \ldots
$$

we see that the number of the commutators $\left[y,{ }_{u} x\right]$ in the second product is equal to $\left|W\left(i_{0}-1, u\right)\right|$, in the first product it is equal to

$$
\sum_{k=1}^{k_{0}-1} E_{n, k}(u, 0)=\sum_{k=1}^{k_{0}-1}\left(\begin{array}{c}
n-k \\
u
\end{array}\right)
$$

Summing the number of the commutators $\left[y,{ }_{u} x\right]$ to the left of $\left[y,{ }_{v} x\right]$ for every $\left[y,{ }_{v} x\right]$ in $P_{n}$, we obtain

$$
f_{n}(u, v)=\sum_{k_{0}=1}^{n-1} \sum_{i_{0} \in W\left(2^{n-k_{0}}-1, v\right)}\left(\left|W\left(i_{0}-1, u\right)\right|+\sum_{k=1}^{k_{0}-1}\left(\begin{array}{c}
n-k \\
u
\end{array}\right)\right) .
$$

Further we use assertion 4) of Theorem 2 and change the indices of summation $k_{0}$ and $i_{0}$ to $m$ and $i$ respectively

$$
\sum_{m=1}^{n-1} \sum_{i \in W\left(2^{n-m}-1, v\right)}|W(i-1, u)|=\sum_{m=1}^{n-1} \sum_{k=1}^{v} \sum_{i=v-k}^{n-m-k}\left(\begin{array}{c}
i \\
u-k+1
\end{array}\right)\left(\begin{array}{c}
n-m-i-1 \\
k-1
\end{array}\right)\left(\begin{array}{c}
i \\
v-k
\end{array}\right) .
$$


Finally, using (16), we obtain

$$
\sum_{m=1}^{n-1} \sum_{i \in W\left(2^{n-m}-1, v\right)} \sum_{k=1}^{m-1}\left(\begin{array}{c}
n-k \\
u
\end{array}\right)=\sum_{m=1}^{n-1} \sum_{k=1}^{m-1}\left(\begin{array}{c}
n-m \\
v
\end{array}\right)\left(\begin{array}{c}
n-k \\
u
\end{array}\right)=\sum_{m=1}^{n-2} \sum_{k=m+1}^{n-1}\left(\begin{array}{l}
m \\
v
\end{array}\right)\left(\begin{array}{l}
k \\
u
\end{array}\right) .
$$

Thus,

$$
f_{n}(u, v)=\sum_{m=1}^{n-1} \sum_{k=1}^{v} \sum_{i=v-k}^{n-m-k}\left(\begin{array}{c}
i \\
u-k+1
\end{array}\right)\left(\begin{array}{c}
n-m-i-1 \\
k-1
\end{array}\right)\left(\begin{array}{c}
i \\
v-k
\end{array}\right)+\sum_{m=1}^{n-2} \sum_{k=m+1}^{n-1}\left(\begin{array}{l}
m \\
v
\end{array}\right)\left(\begin{array}{l}
k \\
u
\end{array}\right) .
$$

Theorem 3 is proved.

Let us note that for any $i>0, k>0,1 \leqslant n \leqslant 3$ the functions $\theta_{n}(i), \mu_{n}(i, k), \mu_{4}(i, k)$ are equal to zero, since $\left(\begin{array}{c}\alpha_{m}(i) \\ 2\end{array}\right)=0$ if $i \geqslant 1$ and $1 \leqslant m \leqslant 2$.

We have the following obvious lemma.

Lemma 2. Suppose $i, k, n \in \mathbb{N}$. Then the following relations hold

1) $\alpha_{n}(i-1)+\alpha_{n}(i)=\alpha_{n+1}(i)$;

2) $\theta_{n}(i)+\left(\begin{array}{c}\alpha_{n}(i) \\ 2\end{array}\right)=\theta_{n+1}(i)$;

3) $\mu_{n}(i, k-1)+\mu_{n}(i, k)=\mu_{n+1}(i, k)$.

Let us prove Theorem 4 . Collecting $x$ and then $y$ in $x y x y$, we obtain

$$
(x y)^{2}=x^{2} y^{2}[y, x][y, x, y] \text {. }
$$

In the identity $(x y)^{3}=(x y)^{2}(x y)$, we replace $(x y)^{2}$ by the right-hand side of (18) and then collect $x, y$ and commutators with weight $\geqslant 2$. So, we have

$$
(x y)^{3}=x^{3} y^{3}[y, x]^{3}[y, x, x][y, x, y]^{5}[y, x, x, y][y, x, y, x] .
$$

It can easily be checked that we get the equalities (18) and (19) by putting $n=2$ and $n=3$ respectively in the formula (12).

Further proof is by induction on $n$. We put $h_{i}=\left[y,{ }_{i} x\right], i=1,2, \ldots$. From the conditions of the theorem it follows that for any natural numbers $i, j$ the commutators $\left[h_{i}, y\right],\left[h_{j}, y\right],\left[h_{i}, h_{j}\right]$ commute with each other and the element $y$.

Let us fix $n \geqslant 3$ and move $x, y$ through every multiplier from (12). We have

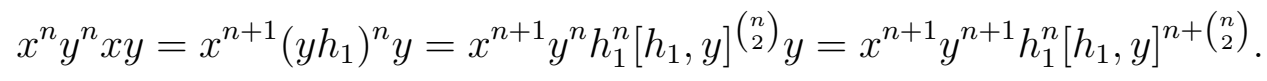

Further, since for every natural numbers $i$ and $m$ the following equalities hold:

$$
h_{i}^{m} x=x\left(h_{i} h_{i+1}\right)^{m}=x h_{i}^{m} h_{i+1}^{m}\left[h_{i+1}, h_{i}\right]^{\left(\begin{array}{c}
m \\
2
\end{array}\right)}, \quad h_{i}^{m} y=y\left(h_{i}\left[h_{i}, y\right]\right)^{m}=y h_{i}^{m}\left[h_{i}, y\right]^{m},
$$

we obtain

$$
h_{i}^{m} x y=x y h_{i}^{m} h_{i+1}^{m}\left[h_{i}, y\right]^{m}\left[h_{i+1}, y\right]^{m}\left[h_{i+1}, h_{i}\right]^{\left(\begin{array}{c}
m \\
2
\end{array}\right)} .
$$

Using relation 1) from Lemma 4 , we get

$$
\prod_{i>0} h_{i}^{\alpha_{n}(i)} h_{i+1}^{\alpha_{n}(i)}=h_{1}^{-n} \prod_{i>0} h_{i}^{\alpha_{n}(i-1)+\alpha_{n}(i)}=h_{1}^{-n} \prod_{i>0} h_{i}^{\alpha_{n+1}(i)}
$$


and

$$
\prod_{i>0}\left[h_{i}, y\right]^{\alpha_{n}(i)}\left[h_{i+1}, y\right]^{\alpha_{n}(i)}=\left[h_{1}, y\right]^{-n} \prod_{i>0}\left[h_{i}, y\right]^{\alpha_{n+1}(i)} .
$$

From (20)-(23) it follows that

$$
x^{n} y^{n} \prod_{i>0} h_{i}^{\alpha_{n}(i)} \cdot x y=x^{n+1} y^{n+1} \prod_{i>0}\left[y,{ }_{i} x\right]^{\alpha_{n+1}(i)} \cdot\left[h_{1}, y\right]^{\left(\begin{array}{c}
n \\
2
\end{array}\right)} \prod_{i>0}\left[h_{i}, y\right]^{\alpha_{n+1}(i)} \cdot \prod_{i>0}\left[h_{i+1}, h_{i}\right]^{\left(\begin{array}{c}
\alpha_{n}(i) \\
2
\end{array}\right)} .
$$

Suppose $n \geqslant 3$ is fixed as above. From definition of the function $\beta_{n}(i, j)$ it follows that

$$
\prod_{i \geqslant 1, j \geqslant 0}\left[y,{ }_{i} x, y,{ }_{j} x\right]^{\beta_{n}(i, j)}=\prod_{j \geqslant 0}\left[h_{1}, y,{ }_{j} x\right]^{\left(\begin{array}{c}
n \\
j+3
\end{array}\right)+\left(\begin{array}{c}
n+1 \\
j+3
\end{array}\right)} \cdot \prod_{i>1, j \geqslant 0}\left[h_{i}, y,{ }_{j} x\right]^{\left(\begin{array}{c}
n+1 \\
i+j+2
\end{array}\right)} .
$$

Since

$$
\begin{aligned}
\prod_{j \geqslant 0}\left[h_{1}, y,{ }_{j} x\right]^{\left(\begin{array}{c}
n \\
j+3
\end{array}\right)+\left(\begin{array}{c}
n+1 \\
j+3
\end{array}\right)} \cdot x y & =x y \prod_{j \geqslant 0}\left[h_{1}, y,{ }_{j} x\right]^{\left(\begin{array}{c}
n \\
j+3
\end{array}\right)+\left(\begin{array}{c}
n+1 \\
j+3
\end{array}\right)}\left[h_{1}, y,{ }_{j+1} x\right]^{\left(\begin{array}{c}
n \\
j+3
\end{array}\right)+\left(\begin{array}{c}
n+1 \\
j+3
\end{array}\right)}= \\
& =x y\left[h_{1}, y\right]^{-\left(\begin{array}{c}
n \\
2
\end{array}\right)-\left(\begin{array}{c}
n+1 \\
2
\end{array}\right)} \prod_{j \geqslant 0}\left[h_{1}, y,{ }_{j} x\right]^{\left(\begin{array}{c}
n \\
j+3
\end{array}\right)+\left(\begin{array}{c}
n+1 \\
j+3
\end{array}\right)+\left(\begin{array}{c}
n \\
j+2
\end{array}\right)+\left(\begin{array}{c}
n+1 \\
j+2
\end{array}\right)}= \\
& =x y\left[h_{1}, y\right]^{-\left(\begin{array}{c}
n \\
2
\end{array}\right)-\left(\begin{array}{c}
n+1 \\
2
\end{array}\right)} \prod_{j \geqslant 0}\left[h_{1}, y, j x\right]^{\left(\begin{array}{c}
n+1 \\
j+3
\end{array}\right)+\left(\begin{array}{c}
n+2 \\
j+3
\end{array}\right)}
\end{aligned}
$$

and

$$
\begin{aligned}
\prod_{i>1, j \geqslant 0}\left[h_{i}, y, j x\right]^{\left(\begin{array}{c}
n+1 \\
i+j+2
\end{array}\right)} \cdot x y & =x y \prod_{i>1, j \geqslant 0}\left[h_{i}, y,{ }_{j} x\right]^{\left(\begin{array}{c}
n+1 \\
i+j+2
\end{array}\right)}\left[h_{i}, y,{ }_{j+1} x\right]^{\left(\begin{array}{c}
n+1 \\
i+j+2
\end{array}\right)}= \\
& =x y \prod_{i>1}\left[h_{i}, y\right]^{-\left(\begin{array}{c}
n+1 \\
i+1
\end{array}\right)} \cdot \prod_{i>1, j \geqslant 0}\left[h_{i}, y,{ }_{j} x\right]^{\left(\begin{array}{c}
n+1 \\
i+j+2
\end{array}\right)+\left(\begin{array}{c}
n+1 \\
i+j+1
\end{array}\right)}= \\
& =x y \prod_{i>1}\left[h_{i}, y\right]^{-\left(\begin{array}{c}
n+1 \\
i+1
\end{array}\right)} \cdot \prod_{i>1, j \geqslant 0}\left[h_{i}, y,{ }_{j} x\right]^{\left(\begin{array}{c}
n+2 \\
i+j+2
\end{array}\right)},
\end{aligned}
$$

we obtain

$$
\prod_{i \geqslant 1, j \geqslant 0}\left[h_{i}, y,{ }_{j} x\right]^{\beta_{n}(i, j)} \cdot x y=x y\left[h_{1}, y\right]^{-\left(\begin{array}{c}
n \\
2
\end{array}\right)} \prod_{i>0}\left[h_{i}, y\right]^{-\alpha_{n+1}(i)} \cdot \prod_{i \geqslant 1, j \geqslant 0}\left[y,{ }_{i} x, y,{ }_{j} x\right]^{\beta_{n+1}(i, j)} .
$$

Finally, suppose $n \geqslant 4$ is fixed. From the definition of the function $\mu_{n}(i, k)$ it follows that

$$
\prod_{i, k>0}\left[\left[y,{ }_{i+1} x\right],\left[y,{ }_{i} x\right],{ }_{k} x\right]^{\mu_{n}(i, k)}=\prod_{i>0}\left[h_{i+1}, h_{i}\right]^{\theta_{n}(i)} \cdot \prod_{i, k>0}\left[h_{i+1}, h_{i},{ }_{k} x\right]^{\mu_{n}(i, k)} .
$$

It is easy to see that for any natural numbers $i, m$ and integer $k \geqslant 0$ we have the equality

$$
\left[h_{i+1}, h_{i},{ }_{k} x\right]^{m} x y=x y\left[h_{i+1}, h_{i},{ }_{k} x\right]^{m}\left[h_{i+1}, h_{i},{ }_{k+1} x\right]^{m} .
$$

Combining that equality and relation 3) from Lemma 2, we obtain

$$
\prod_{i>0}\left[h_{i+1}, h_{i}\right]^{\theta_{n}(i)} x y=x y \prod_{i>0}\left[h_{i+1}, h_{i}\right]^{\theta_{n}(i)} \cdot \prod_{i>0}\left[h_{i+1}, h_{i}, x\right]^{\theta_{n}(i)}
$$


and

$$
\begin{aligned}
\prod_{i, k>0}\left[h_{i}, y,{ }_{k} x\right]^{\mu_{n}(i, k)} \cdot x y & =x y \prod_{i, k>0}\left[h_{i}, y,{ }_{k} x\right]^{\mu_{n}(i, k)} \cdot \prod_{i, k>0}\left[h_{i}, y,{ }_{k+1} x\right]^{\mu_{n}(i, k)}= \\
& =x y \prod_{i>0}\left[h_{i}, y, x\right]^{-\mu_{n}(i, 0)} \cdot \prod_{i, k>0}\left[h_{i}, y,{ }_{k} x\right]^{\mu_{n}(i, k-1)+\mu_{n}(i, k)}= \\
& =x y \prod_{i>0}\left[h_{i}, y, x\right]^{-\theta_{n}(i)} \cdot \prod_{i, k>0}\left[h_{i}, y,{ }_{k} x\right]^{\mu_{n+1}(i, k)} .
\end{aligned}
$$

From (26),(27) it follows that

$$
\prod_{i>0, k \geqslant 0}\left[\left[y,{ }_{i+1} x\right],\left[y,{ }_{i} x\right],{ }_{k} x\right]^{\gamma_{n}(i, k)} \cdot x y=x y \prod_{i>0}\left[h_{i+1}, h_{i}\right]^{\theta_{n}(i)} \cdot \prod_{i, k>0}\left[h_{i+1}, h_{i},{ }_{k} x\right]^{\mu_{n+1}(i, k)} .
$$

To conclude the proof, it remains to use the formulas (24), (25), (28) and relation 2) from Lemma 2. Theorem 4 is proved.

\section{Simplifying the multiple combinatorial sums}

We will compute (multiple) combinatorial sums using mainly the method of coefficients. A brief introduction to the method is set out below, its detailed description can be found in [1].

Let $L$ be the set of a Laurent formal power series over a field $C$ containing only finitely many terms with negative powers. The order of the monomial $c_{k} w^{k}$ is $k$. The order of series $C(w)=\sum_{l} c_{l} w^{l}$ from $L$ is the minimal order of monomials with nonzero coefficient. Let $L_{k}$ denotes a set of series of order $k$. We can introduce in $L$ operations of addition, multiplication, substitution, inversion and differentiation. For $C(w) \in L$ define the operator of formal residue as

$$
\underset{w}{\operatorname{res}} C(w)=c_{-1}
$$

If $A(w)=\sum_{k}^{\infty} a_{k} w^{k}$ be the generating function for the sequence $\left\{a_{k}\right\}$, then $a_{k}=\operatorname{res}_{w} A(w) w^{-k-1}$ for $k=0,1, \ldots$ For example, the binomial coefficients $\left(\begin{array}{l}n \\ k\end{array}\right), n, k=0,1, \ldots$ :

$$
\begin{gathered}
\left(\begin{array}{l}
n \\
k
\end{array}\right)=\operatorname{res}_{w}\left\{\frac{(1+w)^{n}}{w^{k+1}}\right\}=\frac{1}{2 \pi i} \int_{|w|=\varrho} \frac{(1+w)^{n}}{w^{k+1}} d w, \quad \varrho>0, \\
\left(\begin{array}{c}
-n \\
k
\end{array}\right)=\left(\begin{array}{c}
n+k-1 \\
k
\end{array}\right)=\operatorname{res}_{w}\left\{\frac{(1-w)^{-n}}{w^{k+1}}\right\}=\frac{1}{2 \pi i} \int_{|w|=\varrho} \frac{(1-w)^{-n}}{w^{k+1}} d w, \quad 0<\varrho<1 .
\end{gathered}
$$

There are several properties for the residue operator res ([1]-[2]). We list only a two of them, which are used in this article.

Rule 1 (linearity). For any $A(w), B(w) \in L$ and $\alpha, \beta \in \mathbb{C}$

$$
\alpha \underset{w}{\operatorname{res}} A(w)+\beta \underset{w}{\operatorname{res}} B(w)=\underset{w}{\operatorname{res}}(\alpha A(w)+\beta B(w)) .
$$

Rule 2 (substitution). a) For $f(w) \in L_{k}(k \geq 1)$ and $A(w)$ any element of $L$, or b) for $A(w)$ polynomial and $f(w)$ any element of $L$ including a constant

$$
\sum_{k} f(w)^{k} \underset{z}{\operatorname{res}}\left(A(z) z^{-k-1}\right)=A(f(w))
$$


Theorem 5. Suppose $r, n, m \in \mathbb{N}$ and $i \in \mathbb{N}_{0}$. Then we have the $r$-multiple formula of summation

$$
S_{r, m}(n, i)=\sum_{s_{r-1}=1}^{n} \sum_{s_{r-2}=1}^{s_{r-1}} \ldots \sum_{s_{1}=1}^{s_{2}} \sum_{s=1}^{s_{1}}\left(\begin{array}{c}
s \\
i+1
\end{array}\right)^{m}=\left\{\begin{array}{l}
\left(\begin{array}{c}
n+r \\
i+r+1
\end{array}\right), \text { if } m=1 ; \\
\sum_{s=0}^{n-i-1}\left(\begin{array}{c}
r+n-i-s-2 \\
r-1
\end{array}\right)\left(\begin{array}{c}
i+s+1 \\
s
\end{array}\right)^{m}, \text { if } m>1 .
\end{array}\right.
$$

Combining the formula $\left(\begin{array}{c}\alpha_{s}(i) \\ 2\end{array}\right)=\frac{1}{2}\left[\left(\begin{array}{c}s \\ i+1\end{array}\right)^{2}-\left(\begin{array}{c}s \\ i+1\end{array}\right)\right]$ and (31), we get

Corollary 1. The following equality holds:

$$
\mu_{n}(i, k)=\frac{1}{2}\left[-\left(\begin{array}{c}
n \\
i+k+2
\end{array}\right)+\sum_{s=0}^{n-k-i-2}\left(\begin{array}{c}
n-i-s-2 \\
k
\end{array}\right)\left(\begin{array}{c}
i+s+1 \\
i+1
\end{array}\right)^{2}\right] .
$$

Proof of the theorem. First let us show that for any $q, i, r \in \mathbb{N}_{0}$ we have

$$
\sum_{s=1}^{q}\left(\begin{array}{c}
s+r \\
i+r+1
\end{array}\right)=\left(\begin{array}{c}
q+r+1 \\
i+r+2
\end{array}\right)
$$

Indeed, using the representation (29), we obtain

$$
\begin{aligned}
& \sum_{s=1}^{q}\left(\begin{array}{c}
s+r \\
i+r+1
\end{array}\right)=\sum_{s=1}^{q} \underset{w}{\operatorname{res}}\left\{\frac{(1+w)^{s+r}}{w^{i+r+2}}\right\}=\underset{w}{\operatorname{res}}\left\{\frac{(1+w)^{r+1}}{w^{i+r+2}} \cdot \sum_{s=0}^{q-1}(1+w)^{s}\right\}= \\
= & \operatorname{res}_{w}\left\{\frac{(1+w)^{r+1}}{w^{i+r+2}} \cdot \frac{(1+w)^{q}-1}{w}\right\}=\left(\begin{array}{c}
q+r+1 \\
i+r+2
\end{array}\right)-\left(\begin{array}{c}
r+1 \\
i+r+2
\end{array}\right)=\left(\begin{array}{l}
q+r+1 \\
i+r+2
\end{array}\right) .
\end{aligned}
$$

By induction on $r$ and the formula (33), we obtain

$$
S_{r, 1}(n, i)=\sum_{s_{r-1}=1}^{n} \sum_{s_{r-2}=1}^{s_{r-1}} \ldots \sum_{s_{1}=1}^{s_{2}} \sum_{s=1}^{s_{1}}\left(\begin{array}{c}
s \\
i+1
\end{array}\right)=\sum_{s_{r-1}=1}^{n}\left(\begin{array}{c}
s_{r-1}+r-1 \\
i+r
\end{array}\right)=\left(\begin{array}{c}
n+r \\
i+r+1
\end{array}\right) .
$$

Suppose $m>1$. If we replace the index $s$ by $s_{1}-s$ and sum over $s$ and $s_{1}$, we obtain 


$$
\begin{aligned}
& S_{r, m}(n, i)=\sum_{s_{r-1}=1}^{n} \cdots \sum_{s=1}^{s_{1}}\left(\begin{array}{c}
s \\
i+1
\end{array}\right)^{m}= \\
& =\sum_{s_{r-1}=1}^{n} \ldots \sum_{s=0}^{s_{1}-1}\left(\begin{array}{c}
s_{1}-s \\
i+1
\end{array}\right)^{m}= \\
& =\sum_{s_{r-1}=1}^{n} \ldots \sum_{s=0}^{\infty} \operatorname{res}_{x_{1}, \ldots, x_{m}}\left\{\prod_{j=1}^{m} \frac{\left(1-x_{j}\right)^{-i-2}}{x_{j}^{s_{1}-s-i}}\right\}= \\
& =\sum_{s_{r-1}=1}^{n} \ldots \sum_{s_{1}=1}^{s_{2}} \operatorname{res}_{x_{1}, \ldots, x_{m}}\left\{\prod_{j=1}^{m} \frac{\left(1-x_{j}\right)^{-i-2}}{x_{j}^{s_{1}-i}} \sum_{s=0}^{\infty}\left(x_{1} \ldots x_{m}\right)^{s}\right\}= \\
& =\sum_{s_{r-1}=1}^{n} \ldots \sum_{s_{1}=1}^{s_{2}} \operatorname{res}_{x_{1}, \ldots, x_{m}}\left\{\frac{1}{1-x_{1} \ldots x_{m}} \prod_{j=1}^{m} \frac{\left(1-x_{j}\right)^{-i-2}}{x_{j}^{s_{1}-i}}\right\}= \\
& =\sum_{s_{r-1}=1}^{n} \ldots \sum_{s_{1}=0}^{s_{2}-1} \operatorname{res}_{x_{1}, \ldots, x_{m}}\left\{\frac{1}{1-x_{1} \ldots x_{m}} \prod_{j=1}^{m} \frac{\left(1-x_{j}\right)^{-i-2}}{x_{j}^{s_{2}-s_{1}-i}}\right\}= \\
& =\sum_{s_{r-1}=1}^{n} \ldots \sum_{s_{2}=1}^{s_{3}} \operatorname{res}_{x_{1}, \ldots, x_{m}}\left\{\frac{1}{1-x_{1} \ldots x_{m}} \prod_{j=1}^{m} \frac{\left(1-x_{j}\right)^{-i-2}}{x_{j}^{s_{2}-i}} \sum_{s_{1}=0}^{\infty}\left(x_{1} \ldots x_{m}\right)^{s_{1}}\right\}= \\
& =\sum_{s_{r-1}=1}^{n} \ldots \sum_{s_{2}=1}^{s_{3}} \operatorname{res}_{x_{1}, \ldots, x_{m}}\left\{\frac{1}{\left(1-x_{1} \ldots x_{m}\right)^{2}} \prod_{j=1}^{m} \frac{\left(1-x_{j}\right)^{-i-2}}{x_{j}^{s_{2}-i}}\right\} \text {. }
\end{aligned}
$$

Summing similarly $r-2$ more times, we obtain the formula

$$
S_{r, m}(n, i)=\underset{x_{1}, \ldots, x_{m}}{\operatorname{res}}\left\{\frac{\left(1-x_{1} \ldots x_{m}\right)^{-r}}{\left(x_{1} \ldots x_{m}\right)^{n-i}} \prod_{j=1}^{m}\left(1-x_{j}\right)^{-i-2}\right\} .
$$

Furthermore, since

$$
(1-x)^{-m}=\sum_{s=0}^{\infty}\left(\begin{array}{c}
m+s-1 \\
m-1
\end{array}\right) x^{s}, \quad|x|<1,
$$

we have

$$
\begin{aligned}
S_{r, m}(n, i) & =\underset{x_{1}, \ldots, x_{m}}{\operatorname{res}}\left\{\frac{\left(1-x_{1} \ldots x_{m}\right)^{-r}}{\left(x_{1} \ldots x_{m}\right)^{n-i}} \prod_{j=1}^{m}\left(1-x_{j}\right)^{-i-2}\right\}= \\
& =\operatorname{res}_{x_{1}, \ldots, x_{m}}\left\{\frac{1}{\left(x_{1} \ldots x_{m}\right)^{n-i}}\left[\sum_{s=0}^{\infty}\left(\begin{array}{c}
r+s-1 \\
s
\end{array}\right)\left(x_{1} \ldots x_{m}\right)^{s}\right] \prod_{j=1}^{m}\left[\sum_{s=0}^{\infty}\left(\begin{array}{c}
i+s+1 \\
s
\end{array}\right) x_{j}^{s}\right]\right\}= \\
& =\operatorname{res}_{x_{1}, \ldots, x_{m}}\left\{\frac{1}{\left(x_{1} \ldots x_{m}\right)^{n-i}}\left[\sum_{s=0}^{\infty}\left(\begin{array}{c}
r+s-1 \\
s
\end{array}\right)\left(x_{1} \ldots x_{m}\right)^{s}\right]\left[\sum_{s=0}^{\infty}\left(\begin{array}{c}
i+s+1 \\
s
\end{array}\right)^{m}\left(x_{1} \ldots x_{m}\right)^{s}\right]\right\}= \\
& =\operatorname{res}_{z}\left\{\frac{1}{z^{n-i}}\left[\sum_{s=0}^{\infty}\left(\begin{array}{c}
r+s-1 \\
s
\end{array}\right) z^{s}\right]\left[\sum_{s=0}^{\infty}\left(\begin{array}{c}
i+s+1 \\
i+1
\end{array}\right)^{m} z^{s}\right]\right\}= \\
& =\sum_{s=0}^{n-i-1}\left(\begin{array}{c}
r+n-i-s-2 \\
n-i-s-1
\end{array}\right)\left(\begin{array}{c}
i+s+1 \\
i+1
\end{array}\right)^{m}=\sum_{s=0}^{n-i-1}\left(\begin{array}{c}
r+n-i-s-2 \\
r-1
\end{array}\right)\left(\begin{array}{c}
i+s+1 \\
s
\end{array}\right)^{m} .
\end{aligned}
$$


Theorem 5 is proved.

In the following lemma, we show that if $2 i+k+3<n$, and $n$ is prime, then the exponents $\mu_{n}(i, k)$ of the commutators $\left[\left[y,{ }_{i+1} x\right],\left[y,{ }_{i} x\right],{ }_{k} x\right]$ in (12) are divisible by $n$.

Lemma 5. If $n, k, i \in \mathbb{N}$ and $n>k+i+1$, then the following identity holds:

$$
\begin{gathered}
\sum_{s=0}^{n-k-i-2}\left(\begin{array}{c}
n-i-s-2 \\
k
\end{array}\right)\left(\begin{array}{c}
i+s+1 \\
i+1
\end{array}\right)^{2}= \\
=\sum_{s=0}^{\min (i+1, n-k-i-2)}(-1)^{s}\left(\begin{array}{c}
k+s \\
s
\end{array}\right)\left(\begin{array}{c}
n-k-s-1 \\
i-s+1
\end{array}\right)\left(\begin{array}{c}
n \\
k+i+s+2
\end{array}\right) .
\end{gathered}
$$

Proof. We have

$$
S_{n, k, i}=\sum_{s=0}^{n-k-i-2}\left(\begin{array}{c}
n-i-s-2 \\
k
\end{array}\right)\left(\begin{array}{c}
i+s+1 \\
s
\end{array}\right)^{2}=\operatorname{res}_{x, y}\left\{\frac{(1-x y)^{-k-1}(1-x)^{-i-2}(1-y)^{-i-2}}{(x y)^{n-k-i-1}}\right\} .
$$

Changing the variables $x=w /(1+w), y=z /(1+z)$, we obtain

$$
1-x=\frac{1}{1+w}, \quad 1-y=\frac{1}{1+z}, \quad \frac{1}{1-x y}=\frac{(1+w)(1+z)}{1+w+z}
$$

and

$$
d x=\frac{d w}{(1+w)^{2}}, \quad d y=\frac{d z}{(1+z)^{2}}
$$

Therefore,

$$
\begin{aligned}
& S_{n, k, i}=\operatorname{res}_{x, y}\left\{\frac{(1+w)^{k+i+3}(1+z)^{k+i+3}}{(1+w+z)^{k+1}}\left(\frac{(1+w)(1+z)}{w z}\right)^{n-k-i-1} \frac{1}{(1+w)^{2}(1+z)^{2}}\right\}= \\
& =\operatorname{res}_{w, z}\left\{\frac{(1+w+z)^{-k-1}(1+w)^{n}(1+z)^{n}}{(w z)^{n-k-i-1}}\right\}= \\
& =\operatorname{res}_{w, z}\left\{\left(1+\frac{z}{1+w}\right)^{-k-1} \frac{(1+w)^{n-k-1}(1+z)^{n}}{(w z)^{n-k-i-1}}\right\}= \\
& =\operatorname{res}_{w, z}\left\{\sum_{s=0}^{\infty}(-1)^{s}\left(\begin{array}{c}
k+s \\
s
\end{array}\right)\left(\frac{z}{1+w}\right)^{s} \times \frac{(1+w)^{n-k-1}(1+z)^{n}}{(w z)^{n-k-i-1}}\right\}= \\
& =\sum_{s=0}^{n-k-i-2}(-1)^{s}\left(\begin{array}{c}
k+s \\
s
\end{array}\right) \times \underset{w}{\operatorname{res}}\left\{\frac{(1+w)^{n-k-s-1}}{w^{(n-k-i-2)+1}}\right\} \times \operatorname{res}_{z}\left\{\frac{(1+z)^{n}}{z^{(n-k-i-2-s)+1}}\right\}= \\
& =\sum_{s=0}^{n-k-i-2}(-1)^{s}\left(\begin{array}{c}
k+s \\
s
\end{array}\right)\left(\begin{array}{c}
n-k-s-1 \\
n-k-i-2
\end{array}\right)\left(\begin{array}{c}
n \\
(n-k-i-2)-s
\end{array}\right)= \\
& =\sum_{s=0}^{\min (i+1, n-k-i-2)}(-1)^{s}\left(\begin{array}{c}
k+s \\
s
\end{array}\right)\left(\begin{array}{c}
n-k-s-1 \\
i-s+1
\end{array}\right)\left(\begin{array}{c}
n \\
k+i+s+2
\end{array}\right) \text {. }
\end{aligned}
$$

The lemma is proved. 


\section{References}

[1] G. P. Egorychev Integral Representation and the Computation of Combinatorial Sums, volume 59 of Translation of Mathematical Monographs. American Mathematical Society, 1984 .

[2] G. P. Egorychev Method of coefficients: an algebraic characterization and recent applications. Labours Waterloo Workshop on Computer Algebra, Waterloo 5-7 May 2008, Springer Verlag (2009), 1-33. DOI: 10.1007/978-3-642-03562-3

[3] G. P. Egorychev, S. G. Kolesnikov, V. M. Leontiev Two collection formulas, International Algebraic Conference dedicated to the 110th anniversary of Professor A. G. Kurosh (1908-1971), 2018, URL: https://lomonosov-msu.ru/file/event/4623/eid4623_attach_e9e 90e270f009df12cdd7d3771ec9d3395156bb4.pdf

[4] M. Hall Jr. The theory of groups, Macmillian, New York, 1959.

[5] Ph. Hall A contribution to the theory of groups of prime-power order, Proc. London Math. Soc., 36(1932), 1, 29-95. DOI:10.1112/plms/s2-36.1.29

[6] E. F. Krause On the collection process, Proc. Amer. Math. Soc., 15 (1964), 497-504. DOI: $10.2307 / 2034532$

[7] V. M. Leontiev P.Hall's collection formulas with some restrictions on commutator subgroup, August Möbius Contest, 2016, URL:www.moebiuscontest.ru/files/2016/leontiev.pdf (in Russian).

[8] A. I. Skopin Jacobi identity and P. Hall's collection formula in two types of transmetabelian groups, Zap. Nauchn. Sem. Leningr. Otd. Mat. Inst. Akad. Nauk SSSR, 175 (1989), 106112. https://doi.org/10.1007/BF01100121

[9] A. I. Skopin On the collection formula, Zap. Nauchn. Sem. Leningr. Otd. Mat. Inst. Akad. Nauk SSSR, 46 (1974), 59-63 (in Russian). 